TECHNICAL NOTE

\title{
Allele frequencies and diversity parameters of 27 single nucleotide polymorphisms within and across goat breeds
}

\author{
I. CAPPUCCIO,$*$ L. PARISET,$*$ P. AJMONE-MARSAN,†S. DUNNER, O. CORTES,, G. ERHARDT, \\ G. LÜHKEN,§K. GUTSCHER,§S. JOOST, II I. J. NIJMAN, ${ }^{* *}$ J. A. LENSTRA,**P. R. ENGLAND,+† \\ S. ZUNDEL, †+G. OBEXER-RUFF, 㧊A. BEJA-PEREIRA,§§ A. VALENTINI* and THE ECONOGENE \\ CONSORTIUM III \\ *Dipartimento di Produzioni Animali, Università della Tuscia, Via San Camillo de Lellis, 01100 Viterbo, Italy, +Istituto di Zootecnica, \\ Università Cattolica del Sacro Cuore, Via Emilia Parmense, 84, 29100 Piacenza, Italy, ‡Departimento de Producción Animal, \\ Universidad Complutense, 28040 Madrid, Spain, §Institut für Tierzucht und Haustiergenetik, Justus-Liebig-Universität, D-35390 \\ Giessen, Germany, IIEcole Polytechnique Fédérale de Lausanne (EPFL), 1015 Lausanne, Switzerland, ** Faculty of Veterinary Medicine, \\ Utrecht University, Yalelaan 1, 3584 CL Utrecht, the Netherlands, +†Laboratoire d'Ecologie Alpine, Université Joseph Fourier, BP 53,

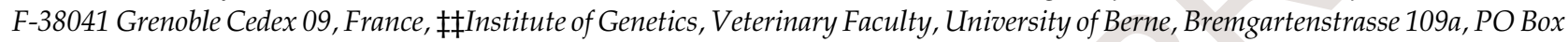 \\ 8466, CH-3001 Berne, Switzerland, §§CIBIO-University of Porto, Campus Agrario de Vairão, 4485-661Vairão, Portugal, IIIhttp:// \\ lasig.epfl.ch/projets/econogene
}

\begin{abstract}
Single-nucleotide polymorphisms (SNPs) are useful markers for biodiversity assessment, linkage analysis, traceability and paternity testing. To date, there are no available SNPs for goat in the NCBI dbSNP database and only a few are reported in the literature. Within the European Union Econogene project, we characterized 27 SNPs in goats using a targeted-gene approach. Polymorphisms were identified in a panel of 16 unrelated individuals belonging to eight different goat breeds selected throughout Europe. Genotypes of 30 goats from each of the eight breeds were determined for all the SNPs characterized and diversity measures were estimated. The caprine SNPs described will be a useful complement to the available genome markers.
\end{abstract}

Keywords: gene diversity, goat, selection, single-nucleotide polymorphisms

Received 24 February 2006; revision accepted 31 March 2006

Ideal genetic markers for population and evolutionary studies should be abundant and distributed widely across the genome, whereas genotyping data must be comparable across laboratories with different scoring methods (Sunnucks 2000). These requirements are fulfilled by the singlenucleotide polymorphisms (SNPs) (Brouillette et al. 2000; Sachidanandam et al. 2001; Shubitowski et al. 2001), which have been shown to be informative suitable for ecological and conservation studies (Vignal et al. 2002; Brumfield et al. 2003; Morin et al. 2004; Seddon et al. 2005), for estimation of parameters such as population history and for inference of relationships (Kuhner et al. 2000; Glaubitz et al. 2003). Furthermore, SNPs are cost-effective for high-throughput

Correspondence: Lorraine Pariset, Fax: +39 761 357434; E-mail: pariset@unitus.it and accurate linkage or association studies (Syvanen 2001; Vitalis et al. 2001; Vignal et al. 2002; Schlötterer 2004). However, in spite of these obvious advantages and their increasing use in human and model organism studies, SNPs have not been employed frequently in studies of nonmodel organisms, which is primarily due to a lack of availability.

Within the Econogene project, we have selected 23 genes involved in key metabolic pathways or potentially relevant for production traits. Primers were designed from goat sequences available at GenBank, or from sequences of related species also available at GenBank. Initially, a panel of 16 unrelated individuals belonging to eight goat breeds from different European regions were used for SNP discovery. This procedure permitted us to avoid ascertainment bias on our heterozygosity estimations by using samples that represented a wide geographical area and 
environments and thus representing the majority of the genetic variation of Goat's gene pool: German Alpine (Germany), Corsican (France), Verata (Spain), Greek goat (Greece), Grigia Molisana (Italy), Baladie (Egypt), Polish fawn improved goat (Polony), Brava (Portugal). The authenticity of the amplification was checked by BLAST searches. A requirement to accept an SNP as authentic (not a sequencing error) and sufficiently polymorphic was that at least two copies of the rarer allele be observed (out of the 32 chromosomes observed).

SNPs were identified in exons, introns and $5^{\prime}$ - or $3^{\prime}$ flanking regions (Table 1). In four genes, two SNPs were identified. Of the $27 \mathrm{SNPs}, 18$ were transitions, eight were transversions and one was a deletion.

The SNPs were subsequently typed by polymerase chain reaction-restriction fragment length polymorphism (PCR-RFLP), PCR single-strand conformation polymorphism (SSCP), SnaPshot, or TaqMan via custom service (K-Bioscience). We genotyped 30 individuals from each of the eight breeds caring that at most only three individuals per farm were analysed. The program POWERMARKER ( $\mathrm{Liu}$ \& Muse 2001) was used to compute the frequencies of the rare allele, the expected heterozygosity $\left(H_{\mathrm{E}}\right)$, the observed heterozygosity $\left(H_{\mathrm{O}}\right)$ (Weir 1996), polymorphic information content (PIC) value (Botstein et al. 1980) and $F$ statistics of genetic differentiation (Weir \& Cockerham 1984) (Table 1), and the program GENECLAss2 (Piry et al. 2004) was used to provide allele frequencies, heterozygotes proportion and Nei's gene diversity for each breed (Table 2).

Expected heterozygosity values ranged from 0.549 (MEG3) to 0.051 (IL2_1) with a mean of 0.358 ; observed heterozygosity values from 0.475 (DES) to 0.040 (IL2_1) with a mean of 0.290. Except one of the two SNPs in the interleukin-2 gene, all SNPs have a frequency of the rare allele higher than $5 \%$ over all breeds and are suitable for genetic analysis. $F_{\mathrm{ST}}$ values are variable within the range of 0.004-0.224, but suggest that breed differentiation by a panel of well-selected SNPs is feasible. The assignment of individuals performed by GENECLAss2 software, using the criterion of Paetkau et al. (1995), assuming default allelic frequency of 0.001 and a threshold of 0.05 , allocated correctly $59.1 \%$ of the individuals using all loci and $61.5 \%$ excluding MSTNG-5 and PRP_IN2 loci, less variable among populations. Assignment of groups of individuals under the same conditions using all loci resulted in $100 \%$ of corrected assignments. Biases can arise when transferring SNP markers across populations (Morin et al. 2004); if these markers are used to analyse non-European breeds, a potential limitation of ascertainment bias should be accounted for.

So far, only few SNPs in goats have been reported in the literature. This study may be a further step towards the exploitation of the vast potential of SNP-based typing within and across goat breeds for a variety of purposes.

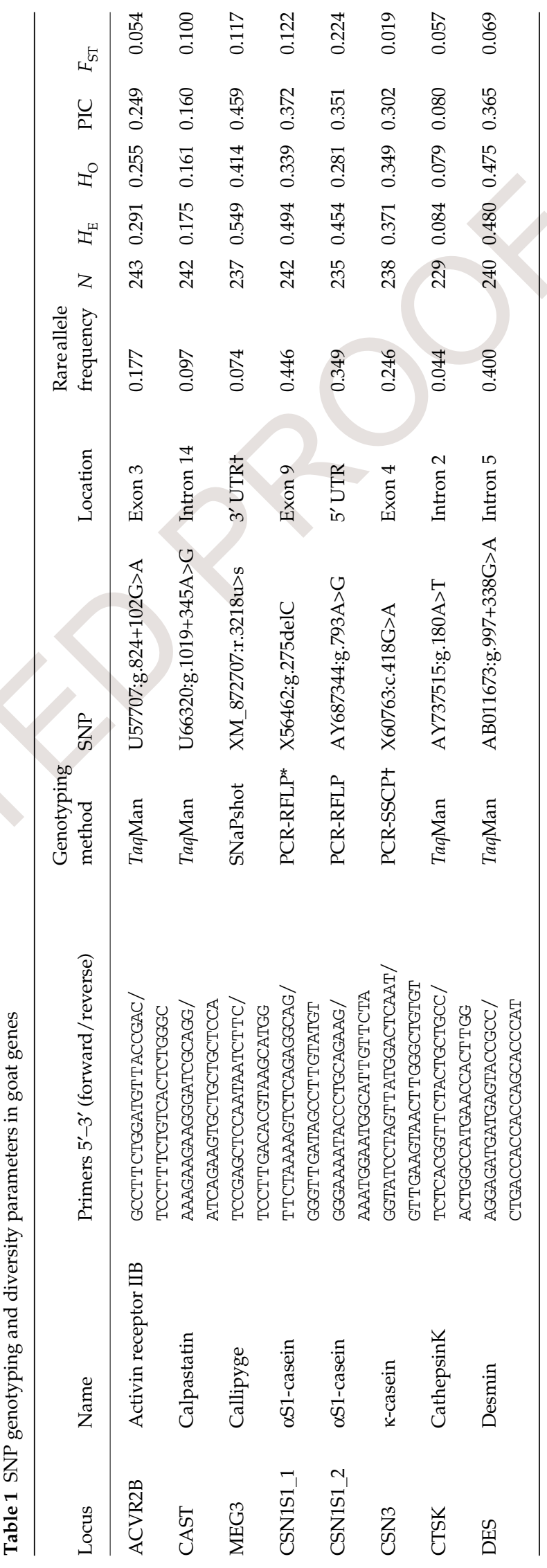




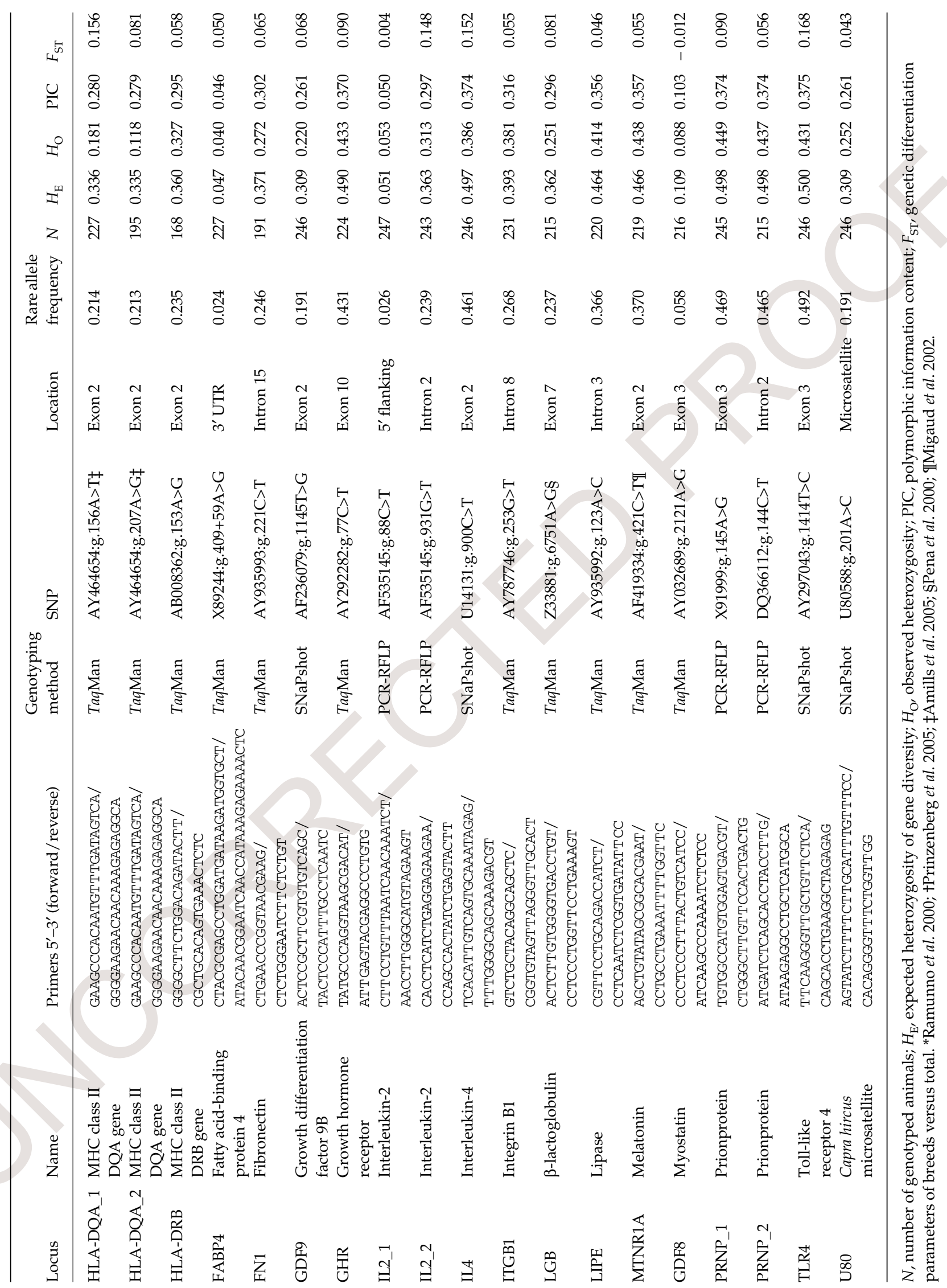

(C) 2006 Blackwell Publishing Ltd, Molecular Ecology Notes, 10.1111/j.1471-8286.2006.01425.x 
Table 2 Allele frequencies, heterozygotes proportion and Nei's gene diversity (Nei 1987) for each locus and each breed

\begin{tabular}{|c|c|c|c|c|c|c|c|c|c|}
\hline \multirow[b]{2}{*}{ Loci } & \multicolumn{9}{|c|}{ Populations } \\
\hline & $\begin{array}{l}\text { German } \\
\text { alpine }\end{array}$ & Verata & Corsican & $\begin{array}{l}\text { Greek } \\
\text { goat }\end{array}$ & $\begin{array}{l}\text { Grigia } \\
\text { molisana }\end{array}$ & Baladie & $\begin{array}{l}\text { Polish fawn } \\
\text { imp. goat }\end{array}$ & Brava & Means \\
\hline \multicolumn{10}{|l|}{ ACVR2Ch353_SNP2 } \\
\hline Frequencies A & 0.774 & 0.933 & 0.8 & 0.717 & 0.919 & 0.677 & 0.817 & 0.95 & 0.823 \\
\hline Frequencies $G$ & 0.226 & 0.067 & 0.2 & 0.283 & 0.081 & 0.323 & 0.183 & 0.05 & 0.177 \\
\hline Heterozygotes proportion & 0.387 & 0.133 & 0.333 & 0.5 & 0.161 & 0.065 & 0.367 & 0.1 & 0.256 \\
\hline \multicolumn{10}{|l|}{ CALPA_ChRev345_SNP2 } \\
\hline Frequencies A & 0.081 & 0.333 & 0.052 & 0.033 & 0.032 & 0.032 & 0.083 & 0.133 & 0.098 \\
\hline Frequencies $\mathrm{G}$ & 0.919 & 0.667 & 0.948 & 0.967 & 0.968 & 0.968 & 0.917 & 0.867 & 0.902 \\
\hline Heterozygotes proportion & 0.161 & 0.4 & 0.103 & 0.067 & 0.065 & 0.065 & 0.167 & 0.267 & 0.162 \\
\hline \multicolumn{10}{|l|}{ CALSNP385R } \\
\hline Frequencies $\mathrm{C}$ & 0.435 & 0.29 & 0.274 & 0.306 & 0.677 & 0.341 & 0.4 & 0.233 & 0.37 \\
\hline Frequencies $\mathrm{G}$ & 0.548 & 0.71 & 0.661 & 0.629 & 0.323 & 0.182 & 0.533 & 0.75 & 0.542 \\
\hline Frequencies T & 0.016 & 0 & 0.065 & 0.065 & 0 & 0.477 & 0.067 & 0.017 & 0.088 \\
\hline Heterozygotes proportion & 0.29 & 0.387 & 0.452 & 0.484 & 0.387 & 0.545 & 0.433 & 0.367 & 0.418 \\
\hline \multicolumn{10}{|l|}{ CSN1_EX9 } \\
\hline Frequencies delC & 0.435 & 0.55 & 0.741 & 0.177 & 0.581 & 0.452 & 0.448 & 0.2 & 0.448 \\
\hline Frequencies wild & 0.565 & 0.45 & 0.259 & 0.823 & 0.419 & 0.548 & 0.552 & 0.8 & 0.552 \\
\hline Heterozygotes proportion & 0.419 & 0.5 & 0.172 & 0.097 & 0.774 & 0.452 & 0.276 & 0 & 0.336 \\
\hline \multicolumn{10}{|l|}{ CSN1S1_5 } \\
\hline Frequencies A & 0.7 & 0.935 & 0.823 & 0.452 & 0.667 & 0.095 & 0.613 & 0.75 & 0.629 \\
\hline Frequencies $\mathrm{G}$ & 0.3 & 0.065 & 0.177 & 0.548 & 0.333 & 0.905 & 0.387 & 0.25 & 0.371 \\
\hline Heterozygotes proportion & 0.267 & 0.129 & 0.355 & 0.258 & 0.333 & 0.19 & 0.387 & 0.3 & 0.277 \\
\hline \multicolumn{10}{|l|}{ CSN3_Ex4 } \\
\hline Frequencies A & 0.823 & 0.645 & 0.839 & 0.806 & 0.79 & 0.795 & 0.71 & 0.633 & 0.755 \\
\hline Frequencies $G$ & 0.177 & 0.355 & 0.161 & 0.194 & 0.21 & 0.205 & 0.29 & 0.367 & 0.245 \\
\hline Heterozygotes proportion & 0.355 & 0.452 & 0.323 & 0.258 & 0.29 & 0.227 & 0.452 & 0.4 & 0.345 \\
\hline \multicolumn{10}{|l|}{ CTSK-G-2 } \\
\hline Frequencies A & 0.065 & 0 & 0 & 0.037 & 0.052 & 0 & 0.173 & 0.033 & 0.045 \\
\hline Frequencies $\mathrm{T}$ & 0.935 & 1 & 1 & 0.963 & 0.948 & 1 & 0.827 & 0.967 & 0.955 \\
\hline Heterozygotes proportion & 0.129 & 0 & 0 & 0.074 & 0.103 & 0 & 0.269 & 0.067 & 0.08 \\
\hline \multicolumn{10}{|l|}{ DESMIN_Ch315_SNP1 } \\
\hline Frequencies A & 0.468 & 0.467 & 0.267 & 0.45 & 0.268 & 0.177 & 0.55 & 0.55 & 0.4 \\
\hline Frequencies $\mathrm{G}$ & 0.532 & 0.533 & 0.733 & 0.55 & 0.732 & 0.823 & 0.45 & 0.45 & 0.6 \\
\hline Heterozygotes proportion & 0.484 & 0.533 & 0.4 & 0.433 & 0.464 & 0.355 & 0.567 & 0.567 & 0.475 \\
\hline \multicolumn{10}{|l|}{ DQA_Ch156_SNP1 } \\
\hline Frequencies $\mathrm{A}$ & 0.967 & 0.466 & 0.759 & 0.897 & 0.946 & 0.893 & 0.672 & 0.685 & 0.786 \\
\hline Frequencies $\mathrm{T}$ & 0.033 & 0.534 & 0.241 & 0.103 & 0.054 & 0.107 & 0.328 & 0.315 & 0.214 \\
\hline Heterozygotes proportion & 0.067 & 0.379 & 0.185 & 0.138 & 0.107 & 0.071 & 0.31 & 0.185 & 0.18 \\
\hline \multicolumn{10}{|l|}{ DQA_Ch207_SNP2 } \\
\hline Frequencies $\overline{\mathrm{A}}$ & 0.111 & 0.238 & 0.06 & 0.431 & 0.24 & 0.288 & 0.024 & 0.262 & 0.207 \\
\hline Frequencies $G$ & 0.889 & 0.762 & 0.94 & 0.569 & 0.76 & 0.712 & 0.976 & 0.738 & 0.793 \\
\hline Heterozygotes proportion & 0.074 & 0 & 0.04 & 0.241 & 0.24 & 0.192 & 0.048 & 0.048 & 0.11 \\
\hline \multicolumn{10}{|l|}{ DRB-G-3 } \\
\hline Frequencies A & 0.407 & 0.079 & 0.222 & 0.194 & 0.25 & 0.094 & 0.364 & 0.115 & 0.216 \\
\hline Frequencies G & 0.593 & 0.921 & 0.778 & 0.806 & 0.75 & 0.906 & 0.636 & 0.885 & 0.784 \\
\hline Heterozygotes proportion & 0.519 & 0.158 & 0.296 & 0.056 & 0.346 & 0.188 & 0.636 & 0.231 & 0.304 \\
\hline \multicolumn{10}{|l|}{ FABP4_Ch1_SNP1 } \\
\hline Frequencies $\mathrm{A}$ & 0.968 & 0.983 & 1 & 1 & 1 & 0.984 & 1 & 0.883 & 0.977 \\
\hline Frequencies $\mathrm{G}$ & 0.032 & 0.017 & 0 & 0 & 0 & 0.016 & 0 & 0.117 & 0.023 \\
\hline Heterozygotes proportion & 0.065 & 0.033 & 0 & 0 & 0 & 0.032 & 0 & 0.167 & 0.037 \\
\hline \multicolumn{10}{|l|}{ FN1 } \\
\hline Frequencies C & 0.286 & 0.341 & 0.08 & 0.36 & 0.341 & 0.357 & 0.188 & 0.042 & 0.249 \\
\hline Frequencies $\mathrm{T}$ & 0.714 & 0.659 & 0.92 & 0.64 & 0.659 & 0.643 & 0.813 & 0.958 & 0.751 \\
\hline Heterozygotes proportion & 0.214 & 0.318 & 0.16 & 0.4 & 0.409 & 0.333 & 0.292 & 0.083 & 0.276 \\
\hline GDFSNP452R & & & & & & & & & \\
\hline Frequencies G & 0.032 & 0.242 & 0.194 & 0.113 & 0.339 & 0.048 & 0.317 & 0.25 & 0.192 \\
\hline Frequencies $\mathrm{T}$ & 0.968 & 0.758 & 0.806 & 0.887 & 0.661 & 0.952 & 0.683 & 0.75 & 0.808 \\
\hline Heterozygotes proportion & 0.065 & 0.161 & 0.194 & 0.161 & 0.355 & 0.097 & 0.433 & 0.3 & 0.221 \\
\hline
\end{tabular}




\begin{tabular}{|c|c|c|c|c|c|c|c|c|c|}
\hline \multirow[b]{2}{*}{ Loci } & \multicolumn{9}{|c|}{ Populations } \\
\hline & $\begin{array}{l}\text { German } \\
\text { alpine }\end{array}$ & Verata & Corsican & $\begin{array}{l}\text { Greek } \\
\text { goat }\end{array}$ & $\begin{array}{l}\text { Grigia } \\
\text { molisana }\end{array}$ & Baladie & $\begin{array}{l}\text { Polish fawn } \\
\text { imp. goat }\end{array}$ & Brava & Means \\
\hline \multicolumn{10}{|l|}{ GHR-G-1a } \\
\hline Frequencies C & 0.645 & 0.638 & 0.37 & 0.517 & 0.5 & 0.897 & 0.481 & 0.444 & 0.561 \\
\hline Frequencies $\mathrm{T}$ & 0.355 & 0.362 & 0.63 & 0.483 & 0.5 & 0.103 & 0.519 & 0.556 & 0.439 \\
\hline Heterozygotes proportion & 0.452 & 0.586 & 0.391 & 0.433 & 0.5 & 0.138 & 0.444 & 0.519 & 0.433 \\
\hline \multicolumn{10}{|l|}{ IL2_5p } \\
\hline Frequencies $C$ & 0.048 & 0 & 0.032 & 0.048 & 0 & 0.032 & 0.048 & 0 & 0.026 \\
\hline Frequencies T & 0.952 & 1 & 0.968 & 0.952 & 1 & 0.968 & 0.952 & 1 & 0.974 \\
\hline Heterozygotes proportion & 0.097 & 0 & 0.065 & 0.097 & 0 & 0.065 & 0.097 & 0 & 0.052 \\
\hline \multicolumn{10}{|l|}{ IL2_In2 } \\
\hline Frequencies $G$ & 0.806 & 0.855 & 0.9 & 0.871 & 0.903 & 0.586 & 0.417 & 0.733 & 0.759 \\
\hline Frequencies $\mathrm{T}$ & 0.194 & 0.145 & 0.1 & 0.129 & 0.097 & 0.414 & 0.583 & 0.267 & 0.241 \\
\hline Heterozygotes proportion & 0.387 & 0.29 & 0.2 & 0.258 & 0.129 & 0.207 & 0.5 & 0.533 & 0.313 \\
\hline \multicolumn{10}{|l|}{ IL4SNP119R } \\
\hline Frequencies C & 0.29 & 0.661 & 0.629 & 0.484 & 0.645 & 0.887 & 0.4 & 0.3 & 0.537 \\
\hline Frequencies $\mathrm{T}$ & 0.71 & 0.339 & 0.371 & 0.516 & 0.355 & 0.113 & 0.6 & 0.7 & 0.463 \\
\hline Heterozygotes proportion & 0.387 & 0.419 & 0.419 & 0.387 & 0.452 & 0.161 & 0.4 & 0.467 & 0.387 \\
\hline \multicolumn{10}{|l|}{ ITGB1-G-2 } \\
\hline Frequencies G & 0.194 & 0.4 & 0.397 & 0.267 & 0.379 & 0.065 & 0.155 & 0.25 & 0.263 \\
\hline Frequencies T & 0.806 & 0.6 & 0.603 & 0.733 & 0.621 & 0.935 & 0.845 & 0.75 & 0.737 \\
\hline Heterozygotes proportion & 0.387 & 0.467 & 0.448 & 0.467 & 0.414 & 0.13 & 0.31 & 0.367 & 0.374 \\
\hline \multicolumn{10}{|l|}{ Lact-G-1 } \\
\hline Frequencies A & 0.107 & 0.192 & 0.306 & 0.167 & 0.518 & 0.25 & 0.103 & 0.25 & 0.237 \\
\hline Frequencies $\mathrm{G}$ & 0.893 & 0.808 & 0.694 & 0.833 & 0.482 & 0.75 & 0.897 & 0.75 & 0.763 \\
\hline Heterozygotes proportion & 0.214 & 0.231 & 0.226 & 0.333 & 0.393 & 0.167 & 0.207 & 0.214 & 0.248 \\
\hline \multicolumn{10}{|l|}{ LIPE-G-1 } \\
\hline Frequencies A & 0.786 & 0.556 & 0.661 & 0.423 & 0.534 & 0.674 & 0.776 & 0.648 & 0.632 \\
\hline Frequencies C & 0.214 & 0.444 & 0.339 & 0.577 & 0.466 & 0.326 & 0.224 & 0.352 & 0.368 \\
\hline $\begin{array}{l}\text { Heterozygotes proportion } \\
\text { mel-G-1 }\end{array}$ & 0.357 & 0.519 & 0.355 & 0.462 & 0.517 & 0.13 & 0.448 & 0.481 & 0.409 \\
\hline Frequencies C & 0.554 & 0.648 & 0.435 & 0.667 & 0.722 & 0.87 & 0.534 & 0.685 & 0.639 \\
\hline Frequencies $\mathrm{T}$ & 0.446 & 0.352 & 0.565 & 0.333 & 0.278 & 0.13 & 0.466 & 0.315 & 0.361 \\
\hline Heterozygotes proportion & 0.321 & 0.407 & 0.613 & 0.519 & 0.407 & 0.261 & 0.517 & 0.407 & 0.432 \\
\hline \multicolumn{10}{|l|}{ MSTNG-5 } \\
\hline Frequencies A & 0.952 & 0.967 & 0.931 & 0.897 & 0.964 & 0.935 & 0.933 & 0.969 & 0.943 \\
\hline Frequencies $\mathrm{G}$ & 0.048 & 0.033 & 0.069 & 0.103 & 0.036 & 0.065 & 0.067 & 0.031 & 0.057 \\
\hline Heterozygotes proportion & 0.032 & 0.067 & 0.069 & 0.138 & 0.071 & 0.13 & 0.133 & 0.063 & 0.088 \\
\hline \multicolumn{10}{|l|}{ PRP_EX3 } \\
\hline Frequencies A & 0.661 & 0.452 & 0.387 & 0.419 & 0.452 & 0.194 & 0.724 & 0.483 & 0.471 \\
\hline Frequencies $\mathrm{G}$ & 0.339 & 0.548 & 0.613 & 0.581 & 0.548 & 0.806 & 0.276 & 0.517 & 0.529 \\
\hline Heterozygotes proportion & 0.484 & 0.516 & 0.581 & 0.516 & 0.581 & 0.258 & 0.345 & 0.3 & 0.448 \\
\hline \multicolumn{10}{|l|}{ PRP_IN2 } \\
\hline Frequencies C & 0.597 & 0.45 & 0.357 & 0.389 & 0.333 & 0.341 & 0.722 & 0.467 & 0.457 \\
\hline Frequencies T & 0.403 & 0.55 & 0.643 & 0.611 & 0.667 & 0.659 & 0.278 & 0.533 & 0.543 \\
\hline Heterozygotes proportion & 0.548 & 0.5 & 0.524 & 0.481 & 0.37 & 0.5 & 0.333 & 0.267 & 0.441 \\
\hline \multicolumn{10}{|l|}{ TL4SNP214R } \\
\hline Frequencies C & 0.258 & 0.613 & 0.452 & 0.484 & 0.306 & 0.742 & 0.35 & 0.867 & 0.509 \\
\hline Frequencies $\mathrm{T}$ & 0.742 & 0.387 & 0.548 & 0.516 & 0.694 & 0.258 & 0.65 & 0.133 & 0.491 \\
\hline Heterozygotes proportion & 0.452 & 0.323 & 0.581 & 0.387 & 0.613 & 0.516 & 0.367 & 0.2 & 0.43 \\
\hline \multicolumn{10}{|l|}{ U80SNP129F } \\
\hline Frequencies A & 0.645 & 0.855 & 0.871 & 0.887 & 0.839 & 0.661 & 0.85 & 0.867 & 0.809 \\
\hline Frequencies C & 0.355 & 0.145 & 0.129 & 0.113 & 0.161 & 0.339 & 0.15 & 0.133 & 0.191 \\
\hline Heterozygotes proportion & 0.387 & 0.29 & 0.258 & 0.226 & 0.258 & 0.097 & 0.3 & 0.2 & 0.252 \\
\hline \multicolumn{10}{|l|}{ All loci } \\
\hline Mean heterozygotes proportion & 0.296 & 0.304 & 0.287 & 0.292 & 0.324 & 0.206 & 0.335 & 0.263 & \\
\hline $\begin{array}{l}\text { Heterozygotes proportion } \\
\text { standard deviation }\end{array}$ & 0.161 & 0.187 & 0.182 & 0.169 & 0.197 & 0.152 & 0.155 & 0.168 & \\
\hline
\end{tabular}




\section{Acknowledgements}

This work was partially supported by the EU Econogene contract QLK5-CT-2001-02461. Major objectives of the project and a list of participants can be found on the website http://lasig.epfl.ch/ projets/Econogene. We would like to thank all the farmers who kindly provided the material.

The content of the publication does not represent necessarily the views of the Commission or its services.

\section{References}

Aitken N, Smith S, Schwarz C, Morin PA (2004) Single nucleotide polymorphism (SNP) discovery in mammals: a targeted-gene approach. Molecular Ecology, 13, 1423-1431.

Amills M, Sulas C, Sanchez A, Bertoni G, Zanoni R, Obexer-Ruff $G$ (2005) Nucleotide sequence and polymorphism of the caprine major histocompatibility complex class II DQA1 (Cahi-DQA1) gene. Molecular Immunology, 42, 375-379.

Bensasson D, Zhang DX, Hartl DL, Hewitt GM (2001) Mitochondrial pseudogenes: evolution's misplaced witnesses. Trends in Ecology \& Evolution, 16, 314-321.

Botstein D, White RL, Skolnick M, Davis RW (1980) Construction of a genetic linkage map in man using restriction fragment length polymorphisms. American Journal of Human Genetics, 32, 314-331.

Brouillette JA, Andrew JR, Venta PJ (2000) Estimate of nucleotide diversity in dogs with a pool-and-sequence method. Mammalian Genome, 11, 1079-1086.

Brumfield RT, Beerli P, Nickerson DA, Edwards SV (2003) The utility of single nucleotide polymorphisms in inferences of population history. Trends in Ecology \& Evolution, 18, 249-256.

Glaubitz JC, Rhodes OE, Dewoody JA (2003) Prospects for inferring pairwise relationships with single nucleotide polymorphisms. Molecular Ecology, 12, 1039-1047.

Kuhner MK, Beerli P, Yamato J, Felsenstein J (2000) Usefulness of single nucleotide polymorphism data for estimating population parameters. Genetics, 156, 439-447.

Liu K, Muse S (2001) POWERMARKER: new genetic data analysis software. Version 3.0. Free program distributed by the author over the internet from www.powermarker.net.

Migaud M, Gavet S, Pelletier J (2002) Partial cloning and polymorphism of the melatonin ${ }_{1 \mathrm{a}}\left(\mathrm{Mel}_{1 \mathrm{a}}\right)$ receptor gene in two breeds of goat with different reproductive seasonality. Reproduction, 124, 59-64.
Morin PA, Luikart G, Wayne RK (2004) SNP workshop Group SNPs in ecology, evolution and conservation. Trends in Ecology \& Evolution, 19, 208-216.

Nei M (1987) Molecular Evolutionary Genetics. Columbia University Press, New York.

Paetkau D, Calvert W, Stirling I, Strobeck C (1995) Microsatellite analysis of population structure in Canadian polar bears. Molecular Ecology, 4, 347-354.

Pena RN, Sánchez A, Folch JM (2000) Characterization of genetic polymorphism in the goat $\beta$-lactoglobulin gene. Journal of Dairy Research, 67, 217-224.

Piry S, Alapetite A, Cornuet J-M, Paetkau D, Baudouin L, Estoup A (2004) GENECLASS2: a software for genetic assignment and firstgeneration migrant detection. Journal of Heredity, 95, 536-539.

Prinzenberg E-M, Gutscher K, Chessa S, Caroli A, Erhardt G (2005) Caprine $\mathrm{K}$-casein (CSN3) polymorphism: new developments in molecular knowledge. Journal of Dairy Science, 88, 1490-1498.

Ramunno L, Cosenza G, Pappalardo M et al. (2000) Identification of the goat CSN1S1F allele by means of PCR-RFLP method. Animal Genetics, 31, 342-343.

Sachidanandam R, Weissman D, Schmidt SC et al. (2001) A map of human genome sequence variation containing 1.42 million single nucleotide polymorphisms. Nature, 409, 928-933.

Schlötterer C (2004) The evolution of molecular markers - just a matter of fashion? Nature Reviews Genetics, 5, 63-69.

Seddon JM, Parker HG, Ostrander EA, Ellegren H (2005) SNPs in ecological and conservation studies, a test in the Scandinavian wolf population. Molecular Ecology, 14, 503-511.

Shubitowski DM, Venta PJ, Douglass CL, Zhou RX, Ewart SL (2001) Polymorphism identification within 50 equine genespecific sequence tagged sites. Animal Genetics, 32, 78-88.

Sunnucks P (2000) Efficient genetic markers for population biology. Trends in Ecology \& Evolution, 15, 199-206.

Syvanen AC (2001) Accessing genetic variation: genotyping single nucleotide polymorphisms. Nature Genetics Reviews, 2, 930-942.

Vignal A, Milan D, San Cristobal M, Eggen A (2002) A review on SNP and other types of molecular markers and their use in animal genetics. Genetics Selection Evolution, 34, 275-305.

Vitalis R, Dawson K, Boursot P (2001) Interpretation of variation across marker loci as evidence of selection. Genetics, 158, 18111823.

Weir BS (1996) Genetic Data Analysis II. Sinauer Associates, Inc, Sunderland, MA.

Weir BS, Cockerham CC (1984) Estimating F-statistics for the analysis of population structure. Evolution, 38, 1358-1370. 


\section{Author Query Form}

\section{Journal: Molecular Ecology Notes}

\section{Article: men_1425.fm}

Dear Author,

During the copy-editing of your paper, the following queries arose. Please respond to these by marking up your proofs with the necessary changes/additions. Please write your answers on the query sheet if there is insufficient space on the page proofs. Please write clearly and follow the conventions shown on the attached corrections sheet. If returning the proof by fax do not write too close to the paper's edge. Please remember that illegible mark-ups may delay publication.

Many thanks for your assistance.

\begin{tabular}{|l|l|l|}
\hline No. & Query & Remarks \\
\hline $\mathbf{1}$ & Please check if postal addresses added here are correct. & \\
\hline $\mathbf{2}$ & $\begin{array}{l}\text { Please confirm change from 'there are in the NCBI dbSNP } \\
\text { database no SNPs available for goat' to 'there are no } \\
\text { available SNPs for goat in the NCBI dbSNP database'. }\end{array}$ & \\
\hline $\mathbf{3}$ & $\begin{array}{l}\text { Vitalis } 2001 \text { has been changed to Vitalis et al. } 2001 \text { so that this } \\
\text { citation matches the one in the list. }\end{array}$ & \\
\hline $\mathbf{4}$ & $\begin{array}{l}\text { Vignal } 2002 \text { has been changed to Vignal et al. 2002 so that this } \\
\text { citation matches the one in the list. }\end{array}$ & \\
\hline $\mathbf{5}$ & $\begin{array}{l}\text { Schlotterer } 2004 \text { has been changed to Schlötterer 2004 so that } \\
\text { this citation matches the one in the list. }\end{array}$ & \\
\hline $\mathbf{6}$ & $\begin{array}{l}\text { Please indicate where Aitken et al. 2004 should be cited in the } \\
\text { text or if this should be removed from the reference list. }\end{array}$ & \\
\hline $\mathbf{7}$ & $\begin{array}{l}\text { Please indicate where Bensasson et al. 2001 should be cited } \\
\text { in the text or if this should be removed from the reference list. }\end{array}$ & \\
\hline
\end{tabular}




\section{Please correct and return this set}

Please use the proof correction marks shown below for all alterations and corrections. If you wish to return your proof by fax you should ensure that all amendments are written clearly in dark ink and are made well within the page margins.

\begin{tabular}{|c|c|c|}
\hline Instruction to printer & Textual mark & Marginal mark \\
\hline Leave unchanged & ... under matter to remain & Stet \\
\hline $\begin{array}{l}\text { Insert in text the matter } \\
\text { indicated in the margin }\end{array}$ & $h$ & $\begin{array}{l}\text { New matter followed by } \\
\boldsymbol{h}\end{array}$ \\
\hline Delete & $\mapsto$ through matter to be deleted & 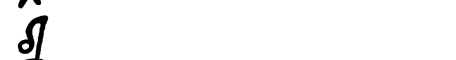 \\
\hline Delete and close up & E through matter to be deleted & \\
\hline $\begin{array}{l}\text { Substitute character or } \\
\text { substitute part of one or } \\
\text { more word(s) }\end{array}$ & $\begin{array}{l}/ \text { through letter or } \mapsto \text { through } \\
\text { word }\end{array}$ & Nंew letter or new word \\
\hline Change to italics & - under matter to be changed & L \\
\hline Change to $\mathrm{c}$ & $\equiv$ under matter to be changed & $\equiv$ \\
\hline Change to $s$ & $=$ under matter to be changed & $=$ \\
\hline Change to bold type & u under matter to be changed & $m$ \\
\hline Change to bold italic & $=$ under matter to be changed & \\
\hline Change to lower case & Encircle matter to be changed & $\#$ \\
\hline Change italic to upright type & (As $a b$ & \\
\hline Insert 'superior' character & $\begin{array}{l}/ \text { through character or } \wedge \text { where } \\
\text { required }\end{array}$ & $\begin{array}{l}y \text { under character } \\
\text { e.g. } 2 y\end{array}$ \\
\hline Insert 'inferior' character & (As above) & $\boldsymbol{L}$ over character e.g. $\boldsymbol{L}$ \\
\hline Insert full $s$ & (As abc & ○ \\
\hline Insert comma & (As a & , \\
\hline Insert single quotation marks & (As abc & y and/or \\
\hline $\begin{array}{l}\text { Insert double quotation } \\
\text { marks }\end{array}$ & (As above) & “6 and/or $y$ \\
\hline Insert hyphen & (As & (4y) \\
\hline Start new paragraph & $\sqrt{-10}$ & $\bar{r}$ \\
\hline No new paragraph & 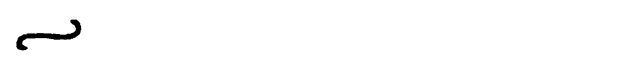 & 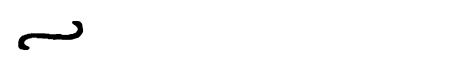 \\
\hline Transpose & 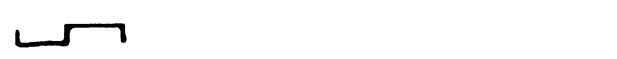 & 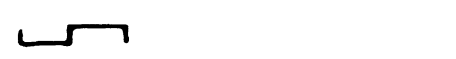 \\
\hline Close up & linking & $=$ \\
\hline Insert space between let & $\boldsymbol{h}$ between letters & $\#$ \\
\hline Insert space between words & $\boldsymbol{h}$ between words affected & \# \\
\hline Reduce space between letters & $\uparrow$ between letters affected & $T$ \\
\hline Reduce space between words & $\tau$ between words affected & \\
\hline
\end{tabular}

